\title{
Luminescent Ruthenium Complex Labels for Correlative Microscopy.
}

Vishwas N. Joshi ${ }^{1}$, Nina H. Pipalia ${ }^{2}$, Eduardo Rosa-Molinar ${ }^{3}$, and Manfred Auer ${ }^{4}$.

1. Nanoprobes, Inc., 95 Horseblock Road, Unit 1, Yaphank, NY 11980.

2. Department of Biochemistry, Weill Cornell Medical College, New York, NY 10065.

3. Microscopy and Analytical Imaging Laboratory, The University of Kansas, Lawrence, KS 66045

4. Molecular Biophysics and Integrated Bioimaging Division, Lawrence Berkeley National Laboratory, Berkeley,CA, USA.

Biological processes are characterized both by their distribution - where they occur in tissues, cells and organelles - and by the structure and interaction of their components. Diffraction-limited light microscopy (LM) and "super-resolution" microscopy (SRM) enable observation of lives cells and tissue details down to hundreds of $\mu \mathrm{m}$ and tens of nm, respectively [1]. Electron microscopy (EM) on the other hand provides much higher resolution [2]. The combination of LM with EM or correlative light and electron microscopy (CLEM), therefore, is a powerful method for correlating dynamic functional information from LM/SRM with static, high-resolution structural information from EM [3].

CLEM reporters have their own merits and shortcomings [4]. Unlike most EM tags, fluorescently labeled colloidal gold nanoparticles (Fl-AuNPs) and semiconductor nanoparticles or "quantum dots" (QDs) are true CLEM probes because they enable direct visualization of exact same targets by both LM and EM without the need for "making them visible" in the EM [4]. Although both Fl-AuNPs and QDs enable multiplexing by CLEM their large hydrodynamic radii reduce resolution by EM while QDs are not fully compatible with many EM sample preparation protocols [4]. Luminescent ruthenium and other platinum group complexes (LMC) have been used as labels for optical microscopy [5]. Also, platinum group metal complexes can be detected with good contrast by scanning EM using a solid-state backscattered electron detector [6]. This makes them ideal molecular CLEM labels (MWs 500-800 Da) for visualization of labeled targets at high spatial resolution. We have discovered that when hydroxylgroups are incorporated onto TCM labels electron-dense silver can be deposited onto labeled targets for their visualization by transmission EM. We herein describe fluorescence and bright-field correlative microscopy with luminescent ruthenium complex (LRuC) labels.

Functionalized polypyridine ligands and their heteroleptic LRuC ruthenium complex labels for crosslinking to primary amine and thiol side-chains of biomacromolecules were prepared following literature procedures [7]. Mouse anti-Thyroglobulin IgG was labeled with LRuC I (Fig. 1) and used to stain commercial human thyroid tumor (BioChain T2235265-5; Fig. 2A - fluorescence). Silver was deposited on LRuC-labeled targets, $\mathrm{pH} \geq 10$ (Fig. 2B - bright-field LM). The fluorescence signal was quenched ( $\sim 15$ fold) after silver deposition (Fig. 2C) but was still detectable. Although LRuCs labels have low quantum yields (0.04 - 0.06), they are photo-stable (laser scanning confocal microscopy data not shown) with large Stokes shifts $(\sim 150 \mathrm{~nm})$ and long-lifetimes (up to $1 \mu \mathrm{s})$ for their imaging by time-resolved techniques for improved signal-to-noise ratios. 
References:

[1] A. M. Sydor et al, Trends in Cell Biology, 25 (2015), 730.

[2] M. P. Oxley et al, Reports on Progress in Physics, 80 (2016), 026101.

[3] P. de Boer et al, Nature Methods, 12 (2016), 503.

[4] E. Brown and P. Verkade, Protoplasma., 244 (2010), 91.

[5] J. N. Demas and B. A. DeGraff, Anal. Chem., 63 (1991), 829A.

[6] J. C. Stokert and G. E. Bertolesi, Micron, 27 (1996), 177.

[7] L. Spiccia et al, Coord. Chem. Rev., 248 (2004), 1329.

[8] Authors wish to thank NIH for financial support - SBIR grant number GM115042.
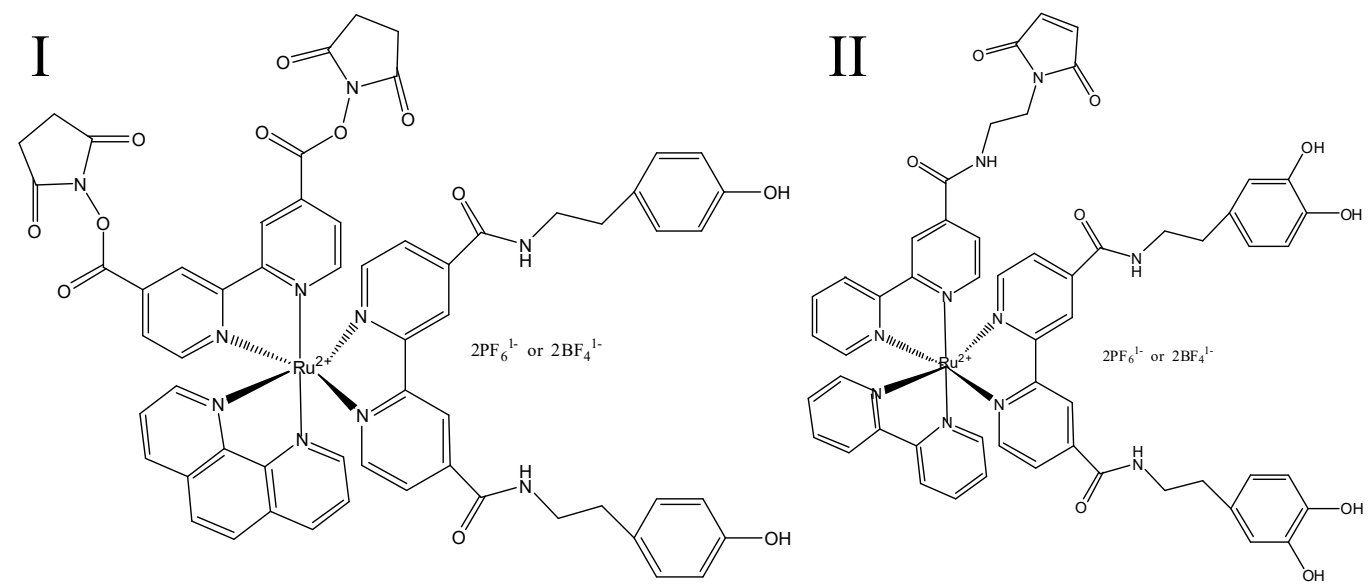

Figure 1. Amine and thiol reactive luminescent ruthenium complex labels

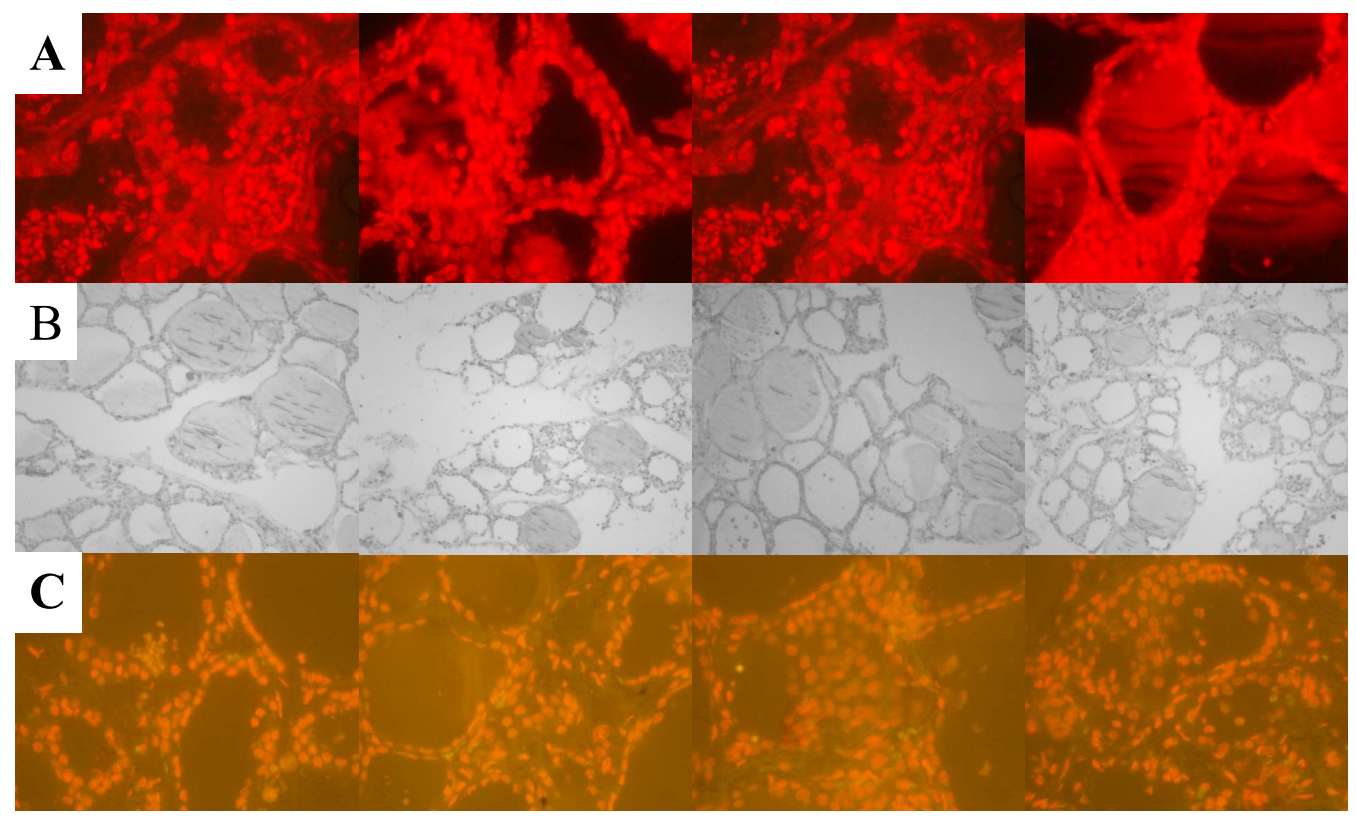

Fig. 2: A) Ru-fluorescence; B) bright-field images following silver deposition at $\mathrm{pH}=10$; and $\mathbf{C}$ ) Silver quenched $\mathrm{Ru}$-fluorescence. A \& C: Excitation $450 \mathrm{~nm} /$ Emission. 610, 40x mag.; B - 10x mag. 